S1 Table. Behaviours of piglets in the home pen after weaning.

\begin{tabular}{ll}
\hline Behaviour & Description \\
\hline Inactive behaviours & \\
Lying & Lying on side or belly without performing any other described behaviour \\
Sitting/kneeling & Sitting or kneeling without performing any other described behaviour \\
Standing & Standing without performing any other described behaviour
\end{tabular}

\title{
Locomotion behaviours
}

Walking

Walking without performing any other described behaviour

\section{Feed-related behaviours}

Exploring feeding

materials

Ingesting feeding

materials

\section{Exploratory behaviours}

Exploring environment

Sniffing or touching feeder without sampling food, touching drinking nipple

Eating or chewing food from feeder or spilled food on floor, drinking from water nipple or eating or chewing wood shavings on the floor

Sniffing, touching, scraping the leg, chewing or rooting (substrate on) floor, toys or any part of the pen, chewing

\section{Social behaviours}

Aggressing pen mates

Head knocking, ramming or pushing a pen mate, with or without biting, piglets involved in a mutual fight

Manipulating pen mates

Rubbing belly of a pen mate with up and down movements of the snout (belly nosing), nibbling, sucking or chewing the tail, ear or any part of the body of a pen mate

Mounting pen mates

Standing on hind legs while having front legs on body of a pen mate

Exploring pen mates

Touching or sniffing any part of the body of a pen mate

\section{Playing behaviours}

Social play

Non-substrate play

Substrate play

Pivoting, rolling, sliding, running around the pen with pen mate(s), gently ramming or nudging pen mates

Pivoting, rolling, sliding, running around the pen alone

Shaking object (straw, toys)

\section{Others}

Maintenance

Rubbing body against objects or pen mates, scratching body with hind legs

Eliminating Defecating or urinating 